\title{
Identification of Building Wall Orientation Angle with Multilook Synthetic Aperture Radars Processing
}

\author{
P. SAmCZYnski ${ }^{a, *}$, K.S. Kulpa ${ }^{a}$, J. MisiurewiCz ${ }^{a}$ And M. SmolarCZYK ${ }^{b}$ \\ ${ }^{a}$ Institute of Electronic Systems, Warsaw University of Technology \\ Nowowiejska 15/19, 00-655 Warsaw, Poland \\ ${ }^{b}$ Przemysłowy Instytut Telekomunikacji S.A., Poligonowa 30, 04-051 Warsaw, Poland
}

\begin{abstract}
A new technique of identifying orientation angles of walls of buildings has been proposed in this paper. The present method is based on multilook processing. The aim of this work is to find the relationships between the received signals reflected from uniform targets (e.g. walls, parts of buildings, etc.) positioned at different orientation angles towards the radar platform. The technique is dedicated for synthetic aperture radars systems. It can be used to extend target identification in synthetic aperture radars images obtained by a single-channel antenna. In this paper, theoretical analysis is presented and verified with simulated radar data.
\end{abstract}

PACS: 84.40.Xb

\section{Introduction}

High quality images of the earth surface can be acquired with several techniques. The most popular one is the optical technique, providing multi-spectral images: from deep infrared to ultraviolet. The optical technique is, however, limited by weather conditions, and with clouds present, it is not possible to make pictures in visual light and ultraviolet, and infrared imaging quality is depreciated significantly. An interesting alternative to optical images is provided by radar images produced with the synthetic aperture radar (SAR) technique. As with optical images, it is possible to produce multispectral images using different wavelengths, typically in the range of 1 to $100 \mathrm{GHz}[1,2]$. The classical single frequency SAR image is a monochrome one, where only the reflection intensity image is created. Synthetic aperture radar is mainly used as a method for creating radar images of the earth areas being observed. Nowadays, several techniques have been developed for target recognition and classification with SAR imaging. The most popular one is based on radar polarimetry [3]. This method made it possible to obtain a number of SAR images by using orthogonally polarized signals. With this technique, it is possible to create e.g. four images using four channels $(\mathrm{HH}, \mathrm{HV}, \mathrm{VV}, \mathrm{VH})$ of the transmitted-received radar antenna. The dedicated signal processing is assigned different colours from the RGB (red - green - blue) colour palette to create different scale images. As a result, a colour SAR image is created, where different colours are assigned to targets characterized by different reflection properties.

* corresponding author; e-mail: psamczyn@elka.pw.edu.pl
Polarimetry techniques are able to recognize and classify e.g. different agriculture areas such as corn, rye, rice fields etc. To improve target recognition, high resolution SAR methods are additionally used. These methods provide high accuracy shapes of agricultural or urban areas (e.g. borders of fields or buildings shapes). However, all of the methods using the polarimetry technique require advanced and expensive polarimetric antennas with multi-channel radar receivers. Because of this, a new method for a single channel SAR radar has been proposed. It is based on multilook processing and takes advantage of directional reflections from different targets, which enables their identification and recognition. The present method is based only on a single polarization antenna; however, it can also be extended in the future as a method for polarimetry techniques to increase target recognition and identification.

In the paper, the first reflection analysis and the preliminary simulation results have been presented. The research was focused mostly on the identification of the building wall orientation angle towards the moving radar platform trajectory. Shapes of huge targets, whose size is larger than a few resolution cells, can be easily recognized in high resolution. In this paper, the authors propose a method which is able to recognize the building wall orientation angle for uniform target sizes comparable to the radar resolution cell.

\section{Multilook processing with the use of RGB colour mapping}

Multilook processing is used mainly as a method to increase the signal to noise ratio (SNR) of SAR images by decreasing Gaussian and speckle noise [3, 4]. The speckle 
noise is a characteristic phenomenon in SAR image creation. However, the authors of this paper show in [5] that multilook processing can also be succesfully applied to improve visualization properties of SAR images by using different colour channels assigned to different multilook channels. In general, multilook SAR processing is based on splitting the full response of a matched filter into $N$ shorter ones, forming $N$ looks at an observed object. This technique can be explained as a division of the real antenna beam $\theta_{\mathrm{H}}$ into $N$ virtual subapertures (see Fig. 1). The match filtering is carried out for each sub-beam separately. As a result, $N$ sub-images are created. The sub-images are non-coherently summed pixel by pixel, and in consequence of this processing the final Multilook SAR image is created.

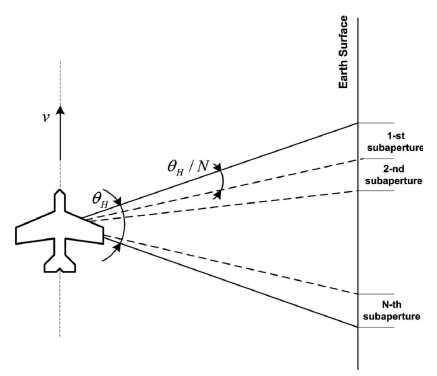

Fig. 1. Multilook SAR geometry.

In the present method, the authors propose to divide the real antenna beam $\theta_{\mathrm{H}}$ into e.g. 3 virtual subapertures and assign one colour from the RGB colour palette to each of the three images thus created. Figure 2 shows an example of the 3-look colour SAR geometry and an example of the plot of one target SAR Doppler frequency.

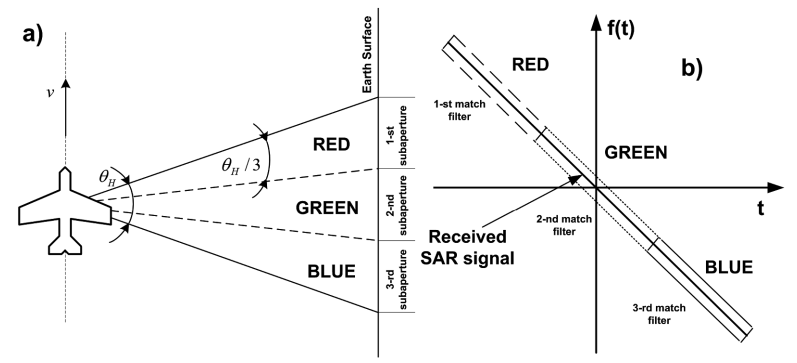

Fig. 2. Three-look colour SAR: (a) geometry, (b) example of the plot of one target SAR Doppler frequency history.

If the scene observed by the radar consists only of a number of prominent points characterized by omnidirectional reflection, and each prominent target can be distinguished in one resolution cell, the final result is the same as in classical multilook processing. The result is visible in white colour as a focused prominent point (all RGB components are summed with the same weight). Differences between multilook SAR images exist when the targets illuminated by the radar are uniform targets consisting of a lot of prominent points located in the same area of one resolution cell. Then the application of the proposed method will result in targets visualized by different colours. The targets will be coloured because of different direction properties of reflection. The analysis of the target reflection for the proposed multilook colour method is presented in the next section of this paper.

\section{Target reflection analysis}

Let us analyze a reflection from a building wall. The geometry for plane $2 \mathrm{D}$ object and for corner reflector (dihedral) modeling the wall is shown in Fig. 3. If the wall is perpendicular to the radar beam and the size of the wall is larger than the beamwidth, then all energy will be scattered back. For a smaller size of the wall, only part of the energy is backscattered towards the radar. In radar community, reflectivity of targets is usually expressed with the radar cross-section (RCS) concept [6]. The radar cross-section of a wall oriented perpendicularly to the beam is equal to $[6]$ :

$$
\mathrm{RCS}=4 \pi H^{2} L^{2} / \lambda^{2},
$$

where $\lambda$ is the wavelength, $H$ is the height and $L$ is the length of the wall.

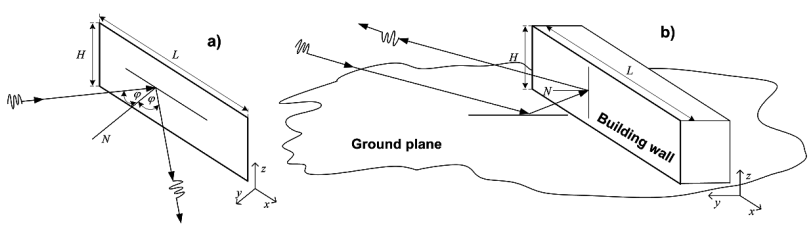

Fig. 3. Geometry of the wave reflection from the wall: (a) 2D model in free space, (b) 3D model with ground reflection.

In most cases, the wall is not perpendicular to the radar beam and consequently the major part of the energy is scattered in another direction, which may be determined with geometrical optics laws. In most cases, we observe not a single wall but more complicated structures composed of several perpendicular facets where multiple reflections are present. One of such examples is a corner reflector. In the simplest situation we observe a single wall on a ground plane. We can also observe structures on roofs of buildings. Such constructions behave as the corner reflector only in the horizontal domain, while in the azimuth domain the energy is side scattered instead of backscattered if the wall is not perpendicular.

When the wall is observed at angle $\varphi$ the backscatter signal in the time domain can then be calculated as [7, 8]:

$$
s(t)=A_{0} \int_{0}^{L \cos \varphi} \exp (\mathrm{j} 2 \pi(t-2 x \tan \varphi / c)) \mathrm{d} x,
$$

where $A_{0}$ is dependent on the total cross-section of the wall (1). Following integration, the final result can be obtained in the following formula: 


$$
s(t)=A_{0} \exp (\mathrm{j} 2 \pi f t) \frac{c[1-\exp (\mathrm{j} 4 \pi f L \sin \varphi / c)]}{j 4 \pi f L \tan \varphi}
$$

The radar cross-section of the wall can then be calculated as a function of incidence angle $\varphi$ :

$$
\begin{aligned}
& \operatorname{RCS}(\varphi)=A_{0} \exp (\mathrm{j} 2 \pi f t) \\
& \quad \times \frac{H^{2}|1-\exp (\mathrm{j} 4 \pi f L \sin \varphi / \lambda)|^{2}}{4 \pi|\tan \varphi|^{2}} .
\end{aligned}
$$

The shape of this function is similar to $\operatorname{sinc}(\sin (x) / x)$. The example cross-section of a $1 \mathrm{~m}$ long wall observed in $\mathrm{X}$ band is presented in Fig. 4. The main sidelobe width is equal to

$$
\Delta \varphi_{a}=\frac{c}{2 f L}=\frac{\lambda}{2 L} .
$$

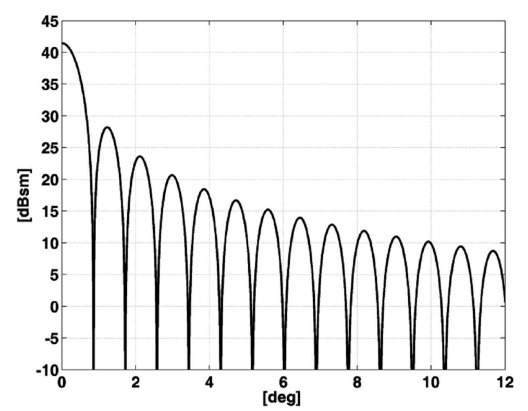

Fig. 4. RCS of the wall versus the observation angle for a $1 \times 1 \mathrm{~m}^{2}$ wall observed by X-band SAR radar.

It is clearly seen that the wall has directional reflectivity, and when observed by a SAR radar, it will provide additional amplitude modulation of the return signal. In such a case, the cross-range resolution of the radar will be decreased.

This effect can be exploited by using multilook SAR processing. In general, multilook processing $[3,4]$ is used to reduce the speckle noise of the final image. In this paper, another application of multilook SAR processing is proposed. In multilook processing, each sub-beam effectively looks in a different azimuth direction. As a result, the incidence angle of the wall is different for each sub-beam, so the energy reflected from the wall will also vary. The change of RCS between sub-beams depends on the angle between the wall and the flight path. The simulated results for walls placed at different angles towards the radar platform are presented in the next section of this paper.

\section{Results}

The current method has been verified with a radar signal simulator [9]. The simulation was performed for a scene which consisted of a uniform wall target built from 200 prominent points. The length of the wall was about $1 \mathrm{~m}$. The simulation was carried out for three scenarios:

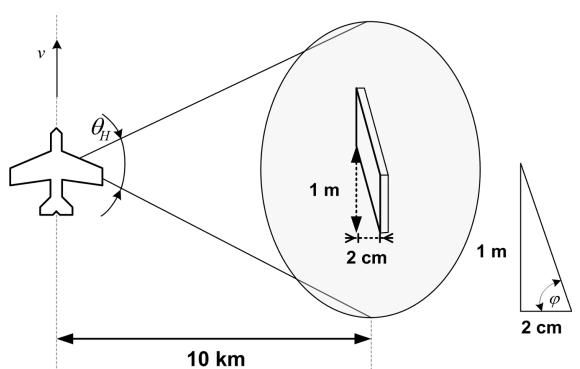

Fig. 5. Simulated SAR scenario geometry for a wall situated at the angle $\varphi=88.8^{\circ}$ towards radar platform flight path.

1. The wall was parallel to the radar platform flight path (wall angle towards radar: $\varphi=0^{\circ}$ ).

2. The wall was situated at the angle $\varphi=88.8^{\circ}$ towards radar platform.

3. The wall was situated at the angle $\varphi=-88.8^{\circ}$ towards radar platform.

Figure 5 illustrates the geometry of the second scenario for the angle $\varphi=88.8^{\circ}$ towards radar platform flight path.

The simulation has been performed for an X-band $(\lambda=$ $0.03 \mathrm{~m}$ ) radar. The radar resolution with focused SAR processing is $c a .1 \mathrm{~m} \times 1 \mathrm{~m}$ in cross-range and range respectively, and resolution with 3-look colour processing is $c a .3 \mathrm{~m} \times 1 \mathrm{~m}$. The illuminated wall shape target was located at a distance of about $10 \mathrm{~km}$.

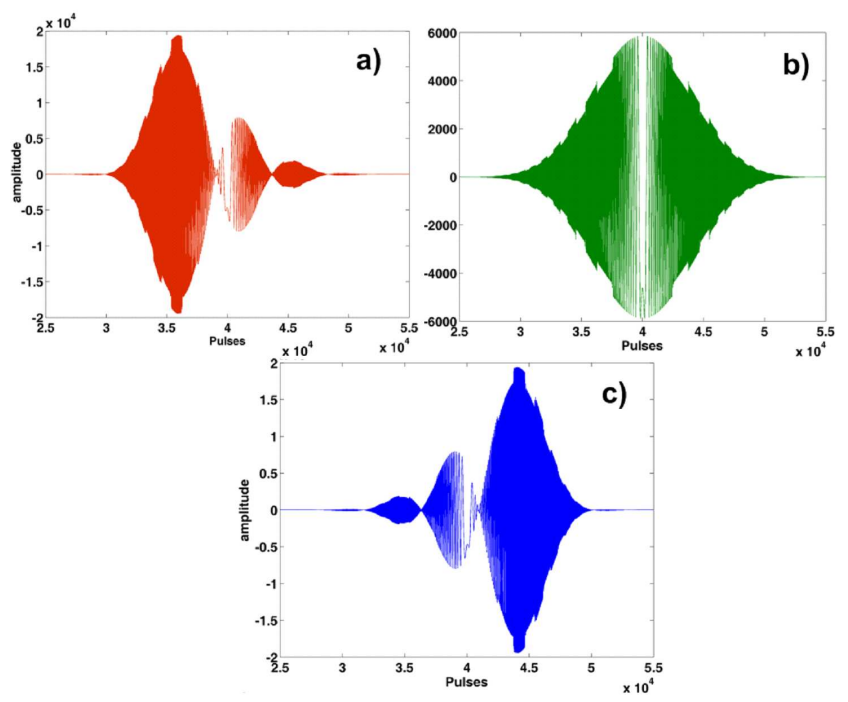

Fig. 6. The real part of SAR signal for 24th range: (a) red channel, (b) green channel, (c) blue channel.

The simulated results are presented in Fig. 6 and Fig. 7.

It can bee seen in Fig. 6 that the received SAR signal envelope changes with the wall orientation angle. The 


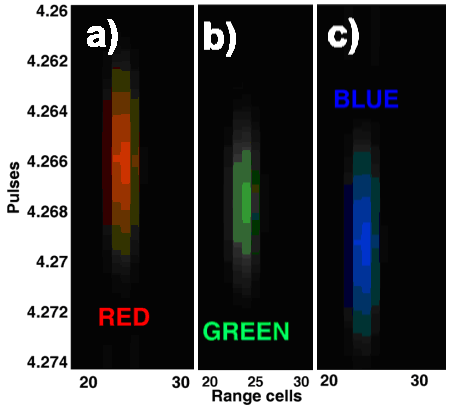

Fig. 7. SAR images created by multilook colour SAR for: (a) red channel, (b) green channel, (c) blue channel.

final result presented in Fig. 7 shows that it is easy to recognize different orientation angles using the proposed multilook colour technique. Moreover, by measuring the power level in each sub-beam the angle of the targets location toward the radar can be estimated.

\section{Conclusions}

Results presented in the paper have shown that the novel multilook colour SAR technique proposed by the authors can be effectively used for wall orientation angle identification. The presented technique extends the classical SAR processing methods for target identification. The method is based on the well known algorithm of multilook processing, commonly implemented in SAR radars. Owing to this it can easily be used in modern radar systems. The application of this method allows the functionality of a classical SAR imaging system to be increased.

\section{Acknowledgments}

This work was partially supported by the Polish Ministry of Science and Higher Education under Commissioned Research Project No R00 018212 for the years 2010-2012 and partially supported by the European Union within the framework of the European Social Fund through the Warsaw University of Technology Development Programme.

\section{References}

[1] G. Franceschetti, R. Lanari, Synthetic Aperture Radar Processing, CRC Press, New York 1999.

[2] J.C. Curlander, R.N. McDonough, Synthetic Aperture Radar Systems and Signal Processing, Wiley, Hoboken 1991.

[3] C. Oliver, S. Quegan, Understanding Synthetic Aperture Radar Images, Artech House, Boston 1997.

[4] P. Samczynski, G. Pietrzyk, Proc. SPIE 6159, 61590 (2006).

[5] A. Gados, A. Gorzelanczyk, M. Mordzonek, P. Samczynski, M. Smolarczyk, K. Kulpa, J. Misiurewicz, in: Proc. of the EuSAR, Vol. 3, Friedrischafen (Germany) 2008 , p. 415.

[6] M. Skolnik, Radar Handbook, 2nd ed., McGraw-Hill, 1990.

[7] K. Kulpa, Habilitation thesis, vol. 164, Publishing House of the Warsaw University of Technology, Warsaw 2008.

[8] J. Misiurewicz, K. Kulpa, Z. Czekala, in: IEE RADAR Conf., Edinburgh 1997, p. 448.

[9] M. Smolarczyk, in: Proc. Int. Radar Symp. 2003, Dresden (Germany), German Institute of Navigation (DGON), Dresden 2003, p. 509. 